Comment on 'On the fusion triple product and fusion power gain of tokamak pilot plants and reactors', by A. Costley

This content has been downloaded from IOPscience. Please scroll down to see the full text.

2017 Nucl. Fusion 57038001

(http://iopscience.iop.org/0029-5515/57/3/038001)

View the table of contents for this issue, or go to the journal homepage for more

Download details:

IP Address: 157.193.64.157

This content was downloaded on 11/04/2017 at 15:40

Please note that terms and conditions apply.

You may also be interested in:

On the fusion triple product and fusion power gain of tokamak pilot plants and reactors

A.E. Costley

Reply to 'Comment "On the fusion triple product and fusion power gain of tokamak pilot plants and reactors"'

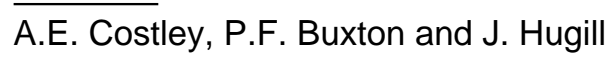

On the power and size of tokamak fusion pilot plants and reactors

A.E. Costley, J. Hugill and P.F. Buxton

From W7-X to a HELIAS fusion power plant: motivation and options for an intermediate-step

burning-plasma stellarator

F Warmer, C D Beidler, A Dinklage et al.

Chapter 1: Overview and summary

M. Shimada, D.J. Campbell, V. Mukhovatov et al.

Relation of vertical stability and aspect ratio in tokamaks

R.D. Stambaugh, L.L. Lao and E.A. Lazarus

ITER: burning plasma physics aspects

B J Green and ITER International Team and Participant Teams

Chapter 6: Steady state operation

C. Gormezano, A.C.C. Sips, T.C. Luce et al.

ADX: a high field, high power density, advanced divertor and RF tokamak

B. LaBombard, E. Marmar, J. Irby et al. 


\title{
Comment
}

\section{Comment on 'On the fusion triple product and fusion power gain of tokamak pilot plants and reactors', by A. Costley}

\author{
W. Biel ${ }^{1}$, K. Lackner ${ }^{2}$, O. Sauter ${ }^{3}$, R. Wenninger ${ }^{2}$ and H. Zohm ${ }^{2}$ \\ ${ }^{1}$ Forschungszentrum Jülich GmbH, Institut für Energie- und Klimaforschung_Plasmaphysik, \\ 52425 Jülich, Germany \\ 2 Max-Planck-Institut für Plasmaphysik, Garching, Germany \\ ${ }^{3}$ Centre de Recherches en Physique des Plasmas, Ecole Polytechnique Federale de Lausanne, \\ Switzerland \\ E-mail:w.biel@fz-juelich.de
}

Received 23 June 2016

Accepted for publication 21 November 2016

Published 23 December 2016

\begin{abstract}
In this comment, we discuss the arguments raised in two recent papers (Costley 2016 Nucl. Fusion 56 066003, Costley et al 2015 Nucl. Fusion 55 033001) on the claimed size independence of fusion power, triple product and fusion gain in tokamak reactors, and we show that all these three quantities actually do depend on the size of the tokamak, when distinguishing between independent input parameters (design parameters) and output quantities, and when taking into account technological limitations.
\end{abstract}

Keywords: tokamaks, fusion reactors, fusion power

In a recent paper [1] the author claims that '.. when the density and beta limit ... are taken into account, the 'triple product' $n T \tau_{\mathrm{E}}$ becomes almost independent of size...', and later on, this argument is used to advocate the use of compact devices to speed up the development of fusion as an energy source. In this comment, we point out that the size argument, in the form quoted above, is misleading.

In [1], the well-known relations for fusion power $P_{\text {fus }}$ and the 'triple product' $n T \tau_{\mathrm{E}}$ (where $n$ is the plasma density, $T$ the plasma temperature and $\tau_{\mathrm{E}}$ the energy confinement time) are combined to yield the equation

$$
n T \tau_{\mathrm{E}} \propto \frac{H^{2} P_{\text {fus }}^{3 / 4}}{\beta_{\mathrm{N}}^{3 / 2} q^{3 / 2} R^{1 / 4}},
$$

and it is argued on the basis of (1) that '...the explicit dependence on size is weak...' '...nT $\tau_{\mathrm{E}}$ does not depend on $A .$. ', and '...there is no explicit dependence on $B_{\mathrm{T}} \ldots$ ' (here, $A=R / a$ denotes the tokamak aspect ratio, $R$ and $a$ the major and minor tokamak radius, $\beta_{\mathrm{N}}$ the normalized plasma beta, $H$ the confinement quality relative to standard $H$ mode [2] and $q$ the safety factor). However, the physical interpretation of equation (1) is flawed due to the fact that the fusion power $P_{\text {fus }}$ is used as in independent input variable that can be held fixed as size is varied. In fact, $P_{\text {fus }}$ is clearly an output that strongly depends on the three design quantities $R, B_{\mathrm{T}}$ and $A$. Indeed, inserting the well-known relation $P_{\text {fus }} \propto \beta_{\mathrm{N}}^{2} B_{\mathrm{T}}^{4} R^{3} /\left(A^{4} q^{2}\right)$ (also given in [1]) into (1), we immediately arrive at

$$
n T \tau_{\mathrm{E}} \propto \frac{H^{2} R^{2} B^{3}}{A^{3} q^{3}} .
$$

This equation, which is also given in [1], clearly shows the strong explicit dependence of the triple product on all three design quantities. We note that a similar argument applies for equation (4) of [1], which is meant to show that also the fusion gain $Q$ is weakly dependent of $R$ and does not depend on $B_{\mathrm{T}}$ and $A$. Also for this argument, $P_{\text {fus }}$ has been used as independent input variable, while the correct use of the design variables $R, B$ and $A$ will lead to a strong dependence 


$$
Q=\frac{1}{\frac{c_{2}}{c_{1}} \frac{q^{3.1} A^{3.53}}{H^{3.23} \beta_{N}^{0.1} B^{3.7} R^{2.7}}-\frac{1}{5}}
$$

as shown in [3], (we note that in [3], a slightly different scaling for $\tau_{\mathrm{E}}$ was used, but that does not affect the generality of the argument).

By holding $P_{\text {fus }}$ fixed while varying $R$, Costley implicitly assumes that $B_{\mathrm{T}}$ is varied with size as $B_{\mathrm{T}} \propto R^{-3 / 4} A q^{1 / 2} \beta_{\mathrm{N}}^{-1 / 2}$. The claimed weak dependence of the triple product and fusion gain on size are fully due to fact that in the presented parameter studies (figures 1 and 2), as well as in an earlier paper [4], Costley reduces the magnetic field when increasing the major radius. Hence, Costley's size argument comes down to the well-known fact that high-field devices can be more compact at same fusion performance. This has been known for many decades and forms the basis for example for the IGNITOR proposal $[5,6]$. However, technology constraints limit both the achievable magnetic field $(B)$ and the maximum power density that the divertor and the first wall can accept [7]. Meaningful reactor designs can only be developed by observing these technological limitations. As an example, ITER has been designed to have the largest magnetic field possible with today's superconducting magnet technology, where the field at the inner leg of the TF coil is limited by the permissible stresses in the coil's structural materials, $\sigma \propto B^{2} / 2 \mu_{0}$ [8], rather than by the properties of the superconductor. Taking into account the radial extent needed for shielding and vacuum vessel, the high on-axis magnetic field value of $B_{\mathrm{T}}=6.5 \mathrm{~T}$ for a 'JET-size' fusion reactor $(R=3 \mathrm{~m}, a=1$ $\mathrm{m}$ ) with $P_{\text {fus }}=200 \mathrm{MW}$, as proposed in figure 5 of [1], could only be generated by TF coils where the maximum field at the inner leg of the coils would significantly exceed the ITER value of 11.6 T. The size of ITER is the minimum necessary to obtain $Q=10$ with an on-axis field of $B_{\mathrm{T}}=5.3 \mathrm{~T}$, when assuming plasma confinement according to the proven standard $\mathrm{H}$ mode conditions with $H=1.0$ [2], providing sufficient space for shielding and for the vacuum safety boundary, and operating at a plasma current according to a safety factor of $q_{95}=3$. These assumed operational conditions for ITER also are compatible with a normalized plasma pressure $\beta_{\mathrm{N}}$ well below the ideal beta limit. Finally, the expected ratio between the power exhausted into the divertor and the major radius, $P_{\text {sep }} / R \sim 15 \mathrm{MW} \mathrm{m}^{-1}$ on ITER is only moderately exceeding the level which has been demonstrated with standard divertor technology in today's tokamaks [9].

Miniaturizing the design of a fusion reactor is highly desirable for cost reduction, but has the consequence that some of the aspects of the device will lie beyond the presently acceptable engineering margins. In addition, the need for a breeding blanket will set limitations to the minimum size of a next-step device aiming at demonstrating all technology needed for a power plant. We note that in [4], these have not been taken into account and also, the physics assumptions exceed substantially the presently achieved ones under stationary conditions. Significant innovations beyond the present knowledge base in both technology and physics are therefore required to open the feasibility of a development path to fusion energy in more compact devices than ITER.

\section{References}

[1] Costley A. 2016 Nucl. Fusion 56066003

[2] ITER Physics Expert Group on Confinement, Transport, ITER Physics Expert Group on Confinement Modelling, Database and ITER Physics Basis 1999 Nucl. Fusion 392175

[3] Zohm H. 2010 Fusion Sci. Technol. 58613

[4] Costley A. et al 2015 Nucl. Fusion 55033001

[5] Coppi B. 1977 Comments Plasma Phys. Control. Fusion 3 47-62

[6] Coppi B. et al 2013 Nucl. Fusion 53104013

[7] Wenninger R. 2015 Proc. 42nd EPS Conf. on Plasma Physics (Lisbon, 2015) (http://ocs.ciemat.es/EPS2015PAP/pdf/ P4.110.pdf)

[8] Duchateau J.-L. 2014 Fusion Eng. Des. 892606

[9] Kallenbach A. et al Nucl. Fusion 55053026 